\title{
CT Evaluation of the shoulder in children with Erb's palsy
}

\author{
R.J.Hernandez and L.Dias \\ Children's Memorial Hospital, Chicago, Illinois, USA
}

\begin{abstract}
The majority of children with traumatic injuries of the brachial plexus recover with only minor sequelae. Some patients develop residual deformity, however. This deformity may consist only of internal rotation contractures, but in some patients subluxation of the humeral head may be present. CT is useful in the evaluation of: the presence or absence of subluxation, deformity of the glenoid and the proper placement of the humeral head in plaster or orthotic devices.
\end{abstract}

Erb's palsy is the most common type of obstetrical injury to the brachial plexus [1]. The majority of patients recover from the injury with only minor sequelae [2], however, in some a residual deformity persists. This deformity may consist only of internal rotation contractures; or in addition some patients may develop subluxation or dislocation of the shoulder. The presence of subluxation of the hu- meral head alters the management of these children $[3,4]$. Although strongly suspected clinically, subluxation of the humeral head may be difficult to demonstrate radiographically.

Previous descriptions of the radiographic appearance of the shoulder in Erb's palsy refer exclusively to its appearance in conventional radiographs $[4,5]$. Although computerized tomography (CT) has been utilized in the evaluation of unstable shoulders [6-8], these reports refer to the nonparalytic shoulder. The purpose of this report is to describe the role of computerized tomography in the evaluation of subluxation of the shoulder in children with Erb's palsy.

\section{Subjects and methods}

Seven children ( 5 female, 2 male) with residual deformity secondary to brachial plexus injuries were examined by CT. Their ages at the time of CT examination varied from 2 months to

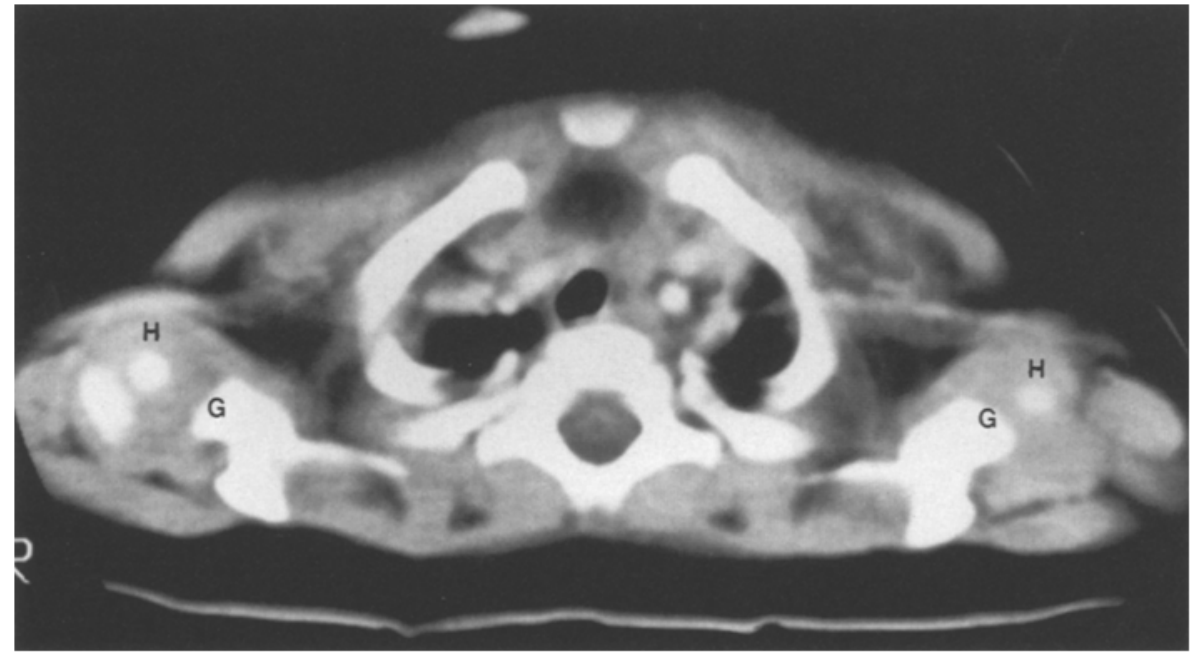

Fig. 1. CT section through the shoulders of a 5-month-old child with normal shoulders. The ossification center of the humeral head $(\mathrm{H})$ is located in the middle of the glenoid fossa $(G)$ 

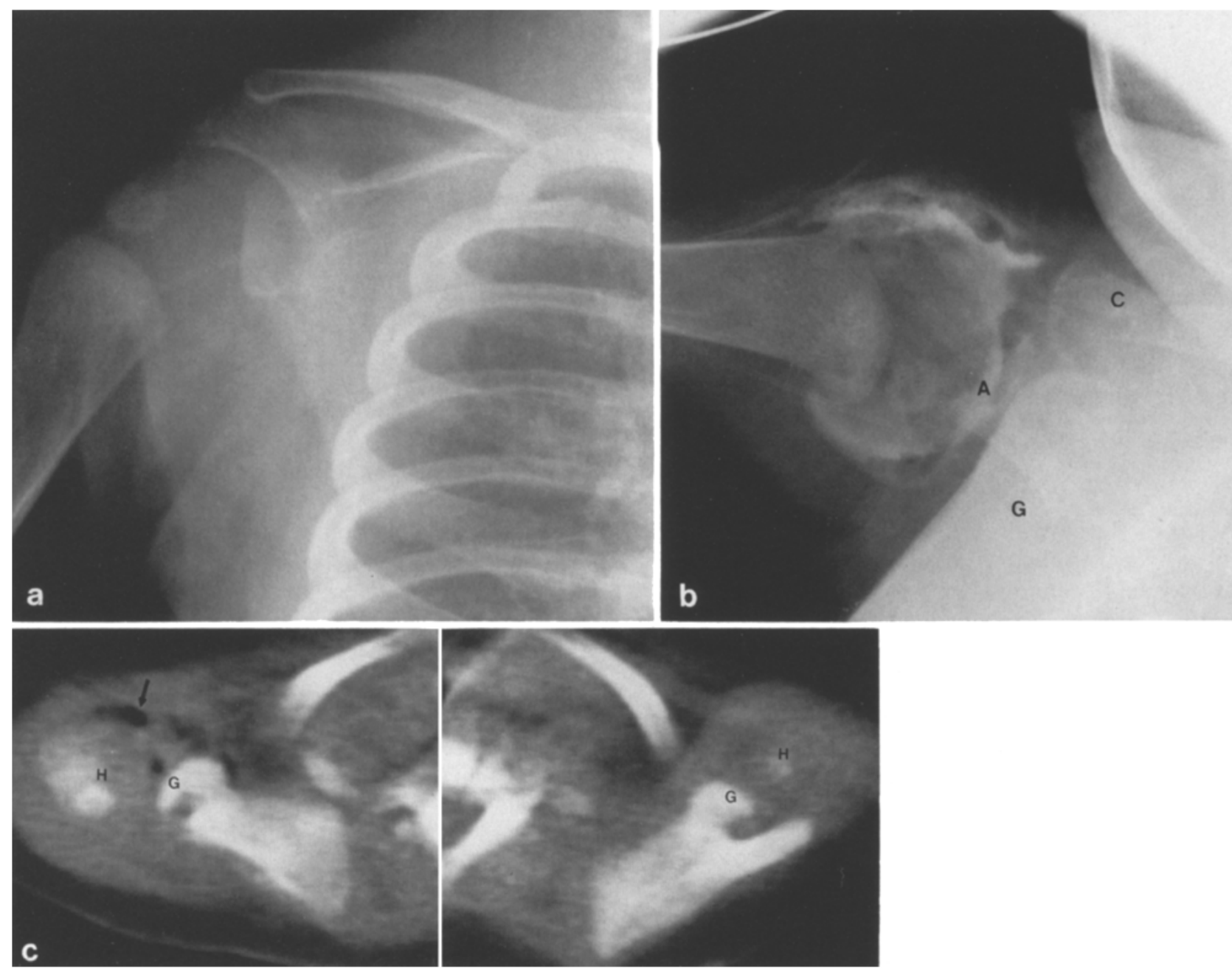

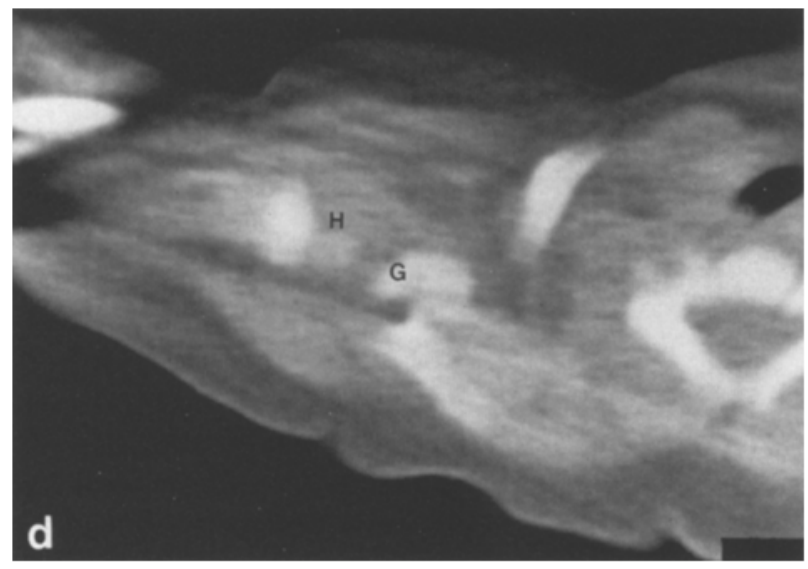

14 years (mean 5 years). All patients except one had right sided paralysis. Conventional radiographs of the shoulder were available for comparison in 6 patients. Only 3 of the 7 patients had axillary views. The CT examinations were performed in five patients with a GE 9800 scanner, the remaining two patients were examined using an EMI 5005 scanner. The patients were examined with their arms by their sides. In addition, 2 patients were examined after the affected extremities had been placed in a brace or cast. Sections were obtained at $10 \mathrm{~mm}$ intervals from
Fig. 2. a Two and a half month old with right Erb's palsy. Anteroposterior view of the right shoulder demonstrates no abnormality. b View of the right shoulder obtained in abduction (after arthrogram) demonstrates normal relationship of the humeral head $(\mathrm{H})$ to the glenoid $(\mathrm{G})$ which was faintly visualized in the radiograph. $\mathrm{C}=$ clavicle. $\mathrm{A}=$ acromion. $\mathrm{c} C \mathrm{~T}$ through the shoulders. Note the posterior relationship of the ossific nucleus of the humeral head $(H)$ to the glenoid $(G)$ on the right side. There is air remaining in the joint space from the arthrogram (arrow). The normal left shoulder demonstrates the normal relationship of the ossific nucleus of the humeral head $(\mathrm{H})$ to the glenoid $(\mathrm{G})$. d After the patient was placed in a splint. The normal relation of the ossific humeral head $(\mathrm{H})$ to the glenoid $(\mathrm{G})$ has been restored

slightly above the acromioclavicular joint to below the glenoid fossa of the scapula.

The relationship of the ossification center of the humeral head to the glenoid, and the deformity of the glenoid were evaluated. Normal shoulders have the ossification center of the humeral head located at the center of the glenoid fossa (Fig.1). A subluxation was defined as present when the ossification center of the humeral head was not located at the midpoint of the glenoid fossa. 


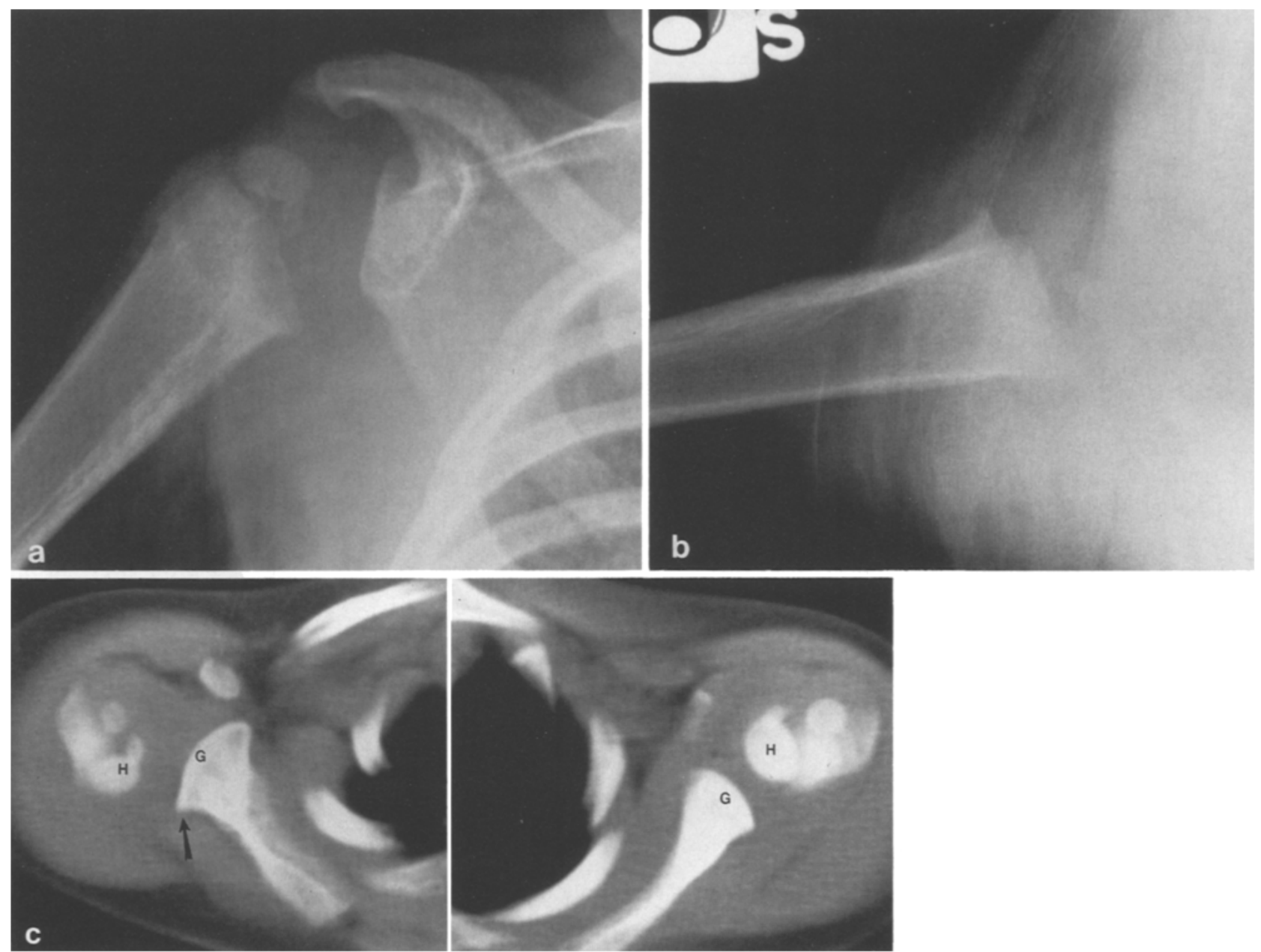

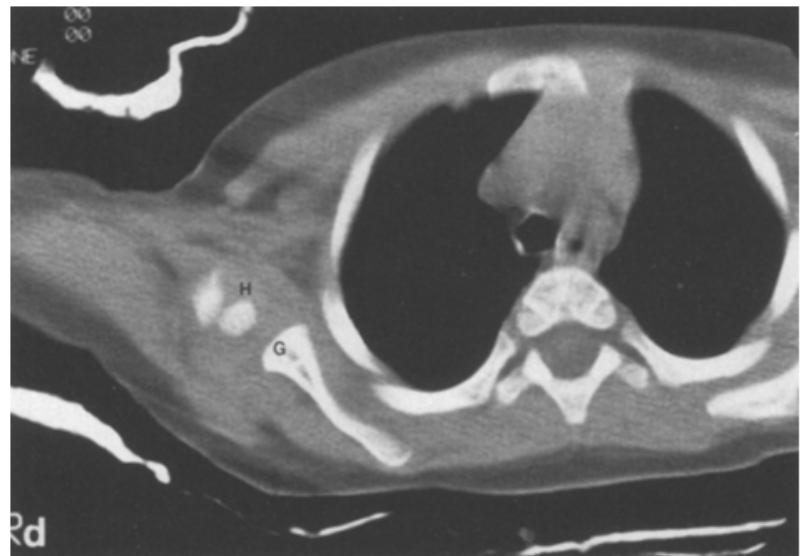

\section{Results}

The findings in the clinical exam, conventional radiographs and CT for each patient are listed in Table 1. Of the 5 patients who had subluxation demonstrated by $\mathrm{CT}$, only one was recognized by conventional radiographic examination of the shoulder (H.A.). Two patients with subluxation
Fig.3. a Frontal radiograph of the right shoulder of an 8-monthold child with right Erb's palsy. The proximal humeral metaphysis is slightly deformed. b Axillary view of the right shoulder demonstrates no subluxation. The suboptimal visualization of the glenoid is due to the young age of the patient, which makes it technically difficult to obtain axillary views. c CT section through the shoulders. The right shoulder demonstrates posterior subluxation of the humeral head $(\mathrm{H})$ and lipping (arrow) of the posterior margin of the glenoid $(G)$. The normal left shoulder demonstrates the normal appearance of the posterior lip of the scapula (arrow) and the normal relationship of the humeral head $(\mathrm{H})$ to the glenoid $(\mathrm{G})$. d CT of the right shoulder after the patient has been placed in a plaster splint. The humeral head $(H)$ is normally related to the glenoid (G)

demonstrated by CT, but not recognized by conventional radiographs, did not have an axillary view of the affected shoulder (Figs. 2, 3).

Only 3 of the 5 patients with subluxation were suspected clinically. In addition, 1 patient (D.S.) with clinical findings of subluxation proved to have a normal shoulder by CT. 
Table 1. Presence or absence of subluxation by clinical examination, conventional radiographs and CT

\begin{tabular}{llll}
\hline & Clinical exam & $\begin{array}{l}\text { Conventional } \\
\text { radiographs }\end{array}$ & CT \\
\hline R.M. & - & $-^{a}$ & + \\
N.B. & + & $-^{a}$ & + \\
M.C. & + & - & + \\
D.B. & + & - & + \\
H.A. & + & + & + \\
B.K. & - & $-{ }^{a}$ & - \\
D.S. & + & $-{ }^{a}$ & - \\
\hline
\end{tabular}

${ }^{a}$ no axillary view obtained

Three of the older patients with subluxation had deformities of the glenoid (Fig.3), which consisted of lipping of the posterior margin of the glenoid.

\section{Discussion}

Trauma at the time of delivery may result in significant injury of the brachial plexus. The majority of children with Erb's palsy will fully recover with minimal or no residual deformity [2]. Nevertheless, some children, because of muscle imbalance, develop persistent deformity of the shoulder consisting of internal rotation contractures, subluxation, or dislocation of the proximal humerus.

Although true dislocation of the humeral head can occur [9], posterior subluxation of the humeral head is more common. This posterior subluxation may be secondary to the position in which the arm is held and to contracture of the internal rotators. This position forces the child to elevate the arm in flexion rather than abduction. Both of these factors force the humeral head to first impinge on and finally slip by the posterior lip of the glenoid fossa [4].

Several surgical procedures have been devised for the correction of the shoulder deformity. These procedures are performed on the soft tissues (i.e., tendon transfers) in the absence of dislocation or subluxation, or on the humerus (osteotomy) when subluxation or dislocation are present $[3,10]$.

Subluxation or dislocation of the humeral head, although strongly suggested by the clinical exam, may be difficult to document radiographically. It is especially difficult in the young child where the humeral head is predominantly cartilaginous and axillary views are difficult to obtain and interpret. The usual radiographic findings in these patients are underdevelopment of the proximal humerus, delayed maturation, or changes in the scapula such as deformity of the glenoid and hypertrophy of the coracoid.
Our study indicates that CT is an excellent imaging modality in the evaluation of subluxation of the shoulder. Not only can the relationship between the proximal humerus and glenoid be visualized, but also, the deformity of the glenoid can be evaluated. Due to the fact that axillary views of the shoulder were not obtained in all patients, no conclusions can be drawn regarding the accuracy of $\mathrm{CT}$ versus conventional radiography. The subluxation in two of our patients was reduced after the arm was placed in abduction in a splint or cast. Since the position of the arm for an axillary view is similar to the position in which the patient is placed in the brace or cast, the subluxation may be reduced in the axillary view as a result of the positioning.

In summary, $\mathrm{CT}$ is useful in evaluating: the relationship between the proximal humerus and scapula, deformity of the scapula, and proper position of the humeral head on the glenoid after the patient has been placed in a splint or brace.

\section{References}

1. Wickstrom J (1962) Birth injuries of the brachial plexus. Treatment of defects of the shoulder. Clin Orthop 23:187

2. Greenwald AG, Schute PC, Shiveley JL (1984) Brachial plexus birth palsy: a 10-year report on the incidence and prognosis. J Pediatr Orthop 4: 689

3. Tachdjian MO (1972) Pediatric orthopedics, vol.2. W.B. Saunders, Philadelphia London Toronto, p 1036

4. DePalma AF (1973) Surgery of the shoulder, 2nd edn. J. B. Lippincott, Philadelphia p 523

5. Sever JW (1916) Obstetric paralysis. Its etiology, pathology, clinical aspects and treatment, with a report of four hundred and seventy cases. Am J Dis Child 12: 541

6. Randelli M, Gambrioli PL (1986) Glenohumeral osteometry by computed tomography in normal and unstable shoulders. Clin Orthop 208:151

7. Seltzer SE, Weissman BN (1985) CT findings in normal and dislocating shoulders. J Can Assoc Radiol 36: 41

8. Danzig L, Resnick D, Greenway G (1982) Evaluation of unstable shoulders by computed tomography. A preliminary study. Am J Sports Med 10: 138

9. Babbitt DP, Cassidy RH (1968) Obstetrical paralysis and dis location of the shoulder in infancy. $J$ Bone Joint Surg [Am] 50: 1447

10. Wickstrom J, Haslam ET, Hutchinson RH (1955) The surgical management of the shoulder following birth injuries of the brachial plexus. J Bone Joint Surg [Am] 37: 27

Received: 4 May 1987; accepted: 17 July 1987

Dr. R. J. Hernandez

Department of Radiology

Section of Pediatric Radiology

University of Michigan Hospitals

C.S. Mott Children's Hospital C 3123

1500 E. Medical Center Drive

Ann Arbor, Michigan 48109-0252

USA 